\title{
Regulation of pre-mRNA Alternative Splicing by the RNA Processing Factor hnRNPL
}

\section{Ganesh S Shankarling*}

Department of Biochemistry, and Biophysics, University of Pennsylvania Perelman School of Medicine, Philadelphia, PA, USA

\section{Significance of Alternative pre-mRNA Splicing}

Sequencing of the human genome has brought into limelight the importance of alternative pre-mRNA splicing to account for the diversity of protein products expressed from a limited repertoire of genes in the genome. Recent studies have indicated that close to $90 \%$ of all human genes are alternatively spliced, with regulation often occurring in a tissue -, and developmental stage - specific manner [1]. Alternative pre-mRNA processing not only provides a unique means for regulating gene expression but is also mis-regulated in a number of human diseases [2]. Therefore, studying the basic mechanism of alternative splicing, and how it regulates normal cell physiology is important for developing novel targets for human disease.

\section{Discovery of hnRNP L}

In our laboratory, we study the regulation of alternative splicing by RNA-binding proteins, and how they impact $\mathrm{T}$ cell function. In particular, my work is focused on the hnRNP L protein, which is the focus of this article. hnRNP stands for heterogeneous nuclear ribonucleic proteins, and they include a family of proteins that bind to RNA, and regulate almost every step of RNA processing, including splicing, mRNA polyadenylation, transport, and translation [3]. The protein composition of the hnRNP complex was first elucidated using monoclonal antibodies against components of the hnRNP complex. The hnRNP L protein was found to be a major component of such immunopurified hnRNP complexes, and was found to be associated with nascent RNA in the nucleus of HeLa cells [4,5]. Cloning and sequence analysis revealed high sequence similarity with hnRNP I/ PTB, another member of the hnRNP complex, and a high degree of evolutionary conservation in vertebrates. A subsequent study revealed that the Drosophila smooth gene is $\sim 50 \%$ identical to the human hnRNP L protein, thus implying an evolutionarily conserved functional role for hnRNP L in RNA metabolism [5].

\section{Regulation of pre-mRNA Alternative Splicing by hnRNP L}

Regulation of pre-mRNA alternative splicing is the best characterized function of hnRNP L in RNA metabolism. The hnRNP L protein contains four RNA Recognition Motifs (RRMs), with each RRM folding into a 4-stranded antiparallel beta sheet connected by two alpha helices. The N-terminal region of hnRNP L is rich in glycines, while RRMs 2, and 3 are connected by a linker region that is rich in prolines. The crystal structure of the RRMs of hnRNP L showed that the RNP1 and RNP2 motifs that are typically the RNA-binding motifs in RRMs are slightly distinct in hnRNP L, due to the fact that the characteristic aromatic residues are replaced by Histidine, Glutamine, and Leucine [6]. Nevertheless, as in other RRMs, the beta sheets in hnRNP L are responsible for binding RNA.

The hnRNP L protein was first reported as a splicing regulator when it was found to stimulate exon inclusion in the human endothelial nitric oxide synthase (eNOS) gene [7].hnRNP L bound to 32 repeats of a CA dinucleotide element in intron 13 of the eNOS mRNA situated downstream of the 5' splice site, and stimulated splicing of the intron. Interestingly, hnRNP L binds to a CA-rich cluster in intron 6 of its own pre-mRNA, and stimulates inclusion of a downstream poison exon resulting in nonsense-mediated decay (NMD) of the hnRNP L mRNA, and down regulation of protein expression [8]. This auto regulatory feedback loop serves to finely balance the homeostatic levels of the hnRNP L protein. Additional studies have revealed several other mRNAs wherein hnRNP L binding to introns or exons stimulate splicing or exon inclusion in HeLa cells [9]. However, the exact mechanism by which hnRNP L stimulates splicing is unknown.

\section{Regulation of CD45 Alternative Splicing by hnRNP L}

Perhaps the best-characterized mRNA target of hnRNP L is the CD45 pre-mRNA, which has led to a deep understanding of the mechanistic basis of splicing regulation by hnRNP L. The CD45 premRNA encodes a transmembrane protein tyrosine phosphatase that functions as a positive regulator in $\mathrm{T}$ cell antigen receptor signaling.The CD45 gene contains 33 exons, of which exons 4, 5, and 6 are alternate exons. In naïve T cells, longer isoforms of CD45 mRNA are expressed which contain variable combinations of exons 4,5 , and 6 . By contrast, in $\mathrm{T}$ cells stimulated with antigen (activated $\mathrm{T}$ cells), alternate exons 4,5 , and 6 are skipped resulting in expression of the shorter, inactive, CD45 isoform (R0). The activation-induced downregulation of CD45 phosphatase activity is important for the termination of $\mathrm{T}$ cell response to antigen and hence critical for T cell homeostasis [10]. Using a clonal line of Jurkat cells, termed JSL1 that mimics the activation-induced alternative splicing pattern of CD45 mRNA observed in primary $\mathrm{T}$ cells, we showed that hnRNP L binds to a signal-responsive sequence element in each of the three alternate exons of the CD45 pre-Mrna [11]. Binding of hnRNP $\mathrm{L}$ to this exonic splicing silencer sequence (termed ARS for "activation responsive sequence"), leads to exon skipping in resting and PhorbolMyristate Acetate (PMA)-stimulated JSL1 cells. Initial efforts in investigating the mechanism of spliceosome regulation by hnRNP L showed that hnRNP L functions to repress splicing by stalling the stalling the spliceosome after the ATP-dependent addition of U1 and U2 snRNPs to the flanking splice sites of exon 4 [12]. Further research has led to the identification of hnRNP A1 as the protein that mediates most, if not all of the splicing repression function of hnRNP L. Using in vitro, and minigene assays, we showed that hnRNP $\mathrm{L}$ recruits hnRNP A1 at the 3' end of the CD45 exon 4, and induces

*Corresponding author: Ganesh S Shankarling, Department of Biochemistry, and Biophysics, University of Pennsylvania Perelman School of Medicine, Philadelphia, PA, USA, E-mail: ganeshs@mail.med.upenn.edu

Received October 01, 2013; Accepted October 02, 2013; Published October 06 2013

Citation: Shankarling GS (2013) Regulation of pre-mRNA Alternative Splicing by the RNAProcessing Factor hnRNPL. J Biomol Res Ther 2: e118. doi: 10.4172/21677956.1000e118

Copyright: ( 2013 Shankarling GS. This is an open-access article distributed under the terms of the Creative Commons Attribution License, which permits unrestricted use, distribution, and reproduction in any medium, provided the original author and source are credited. 
extended base-pairing interactions with U1 snRNA, leading to a block in spliceosome assembly, and exon inclusion [13]. In complimentary studies, we used MS2-tethering assays to delineate the domains of hnRNP L that are required for repressing exon inclusion. These studies revealed that the linker region of hnRNP L - and to a smaller extent - the glycine-rich $\mathrm{N}$-terminal region are required for splicing repression, while any of the RRMs were dispensable for silencing exon inclusion. The glycine-rich and linker regions of hnRNP L were not sufficient to confer exon repression, and required the specific presence of at least one RRM to confer repression [14]. In summary, data from our laboratory using CD45 pre-mRNA as model has led to critical insights into the mechanism by which splicing regulators manipulate dynamic interactions of the components of the spliceosome, leading to regulation of splice site choice. In T cells, regulation of alternative splicing by hnRNPL is not limited to CD45. Knockout mice carrying deletion of hnRNP L specifically in T cells exhibited defects in T cell differentiation, proliferation, and migration [15]. These studies indicate that hnRNP L regulates processing of a broad range of mRNAs critical to $\mathrm{T}$ cell function. Further studies investigating the transcriptome-wide RNA target regulation of hnRNP L will be crucial in understanding the central of hnRNP $\mathrm{L}$ in regulating $\mathrm{T}$ cell physiology, and function.

\section{Regulation of Alternative Splicing by hnRNP L in Diverse Tissues}

Though our laboratory has primarily studied the function of hnRNP L in regulating CD45 splicing, it is known that this protein is ubiquitously expressed and controls expression of many other genes. In neuronal cells, hnRNP L binds to $\mathrm{Ca}^{2+} /$ calmodulin-dependent protein kinase IV (CAMKIV)-responsive RNA element (CARRE1) that consists short CA-repeat motifs with adjacent A/C-rich sequence. In this model, hnRNP L binds to the CARRE motif present upstream of the $3^{\prime}$ splice site of the stress axis-regulated exon (STREX) of the $\mathrm{Ca}^{2+} /$ voltage-sensitive Slo potassium channel gene, and represses exon inclusion in a CAMKIV, and depolarization-dependent manner [16]. Additionally, hnRNP L is phosphorylated on Serine 513 by CaMKIV upon depolarization, leading to increased binding of hnRNP L to the CARRE1 element, blocking of U2AF65 binding to the $3^{\prime}$ splice site, and repression of exon inclusion upon depolarization [17].

Interestingly, phosphorylated hnRNP L plays a key role in tumorigenic capacity in non-small cell lung cancer by regulating RNA processing of the caspase9 pre-mRNA. In this model, hnRNP $\mathrm{L}$ is specifically phosphorylated on Serine 52 in non-small cell lung cancer cells [18].Phospho-hnRNP L binds to variable exon 3 in the caspase 9 pre-mRNA leading to the preferential expression of the antiapoptotic form of caspase 9, thereby contributing to tumor initiation, and progression. Regulation of caspase 9 RNA processing by hnRNP L provides an elegant example by which hnRNPs can contribute to cancer, and provides an opportunity to develop novel strategies to treat such disease.

\section{Future Studies}

As described above, hnRNP L plays a key role in regulating alternative splicing of pre-mRNA targets in several tissues. However, apart from CD45, a deeper understanding of the mechanism by which hnRNP L regulates spliceosome assembly, and function is lacking. Such mechanistic insights into hnRNP L function are needed to better understand its role in regulating normal cell physiology, and also to develop novel drug targets. In addition to regulating alternative splicing, hnRNP L is also known to influence other aspects of RNA processing such as mRNA polyadenylation, export, and stability [9]. However, the mechanisms by which hnRNP L influences aspects of RNA processing other than splicing are not clear. Therefore, future work aimed at functionally characterizing the role of hnRNP $\mathrm{L}$ in regulation of diverse aspects of RNA processing is important to fully realize its central role in regulation the normal cell physiology, and its contribution to the etiology of human disease.

\section{Reference}

1. Wang ET, Sandberg R, Luo S, Khrebtukova I, Zhang L, et al. (2008) Alternative isoform regulation in human tissue transcriptomes. Nature 456: 470-476.

2. Cooper TA, Wan L, Dreyfuss G (2009) RNA and disease. Cell 136: 777-793.

3. Han SP, Tang YH, Smith R (2010) Functional diversity of the hnRNPs: past, present and perspectives. Biochem J 430: 379-392.

4. Pinol-Roma S, Swanson MS, Gall JG, Dreyfuss G (1989)A novel heterogeneous nuclear RNP protein with a unique distribution on nascent transcripts. J Cell Biol 109: 2575-2587

5. Zur Lage P, Shrimpton AD, Flavell AJ, Mackay TF, Brown AJ (1997) Genetic and molecular analysis of smooth, a quantitative trait locus affecting bristle number in Drosophila melanogaster. Genetics 146: 607-618.

6. Zhang W, Zeng F, Liu Y, Zhao Y, Lv H, et al. (2013) Crystal Structures and RNAbinding Properties of the RNA Recognition Motifs of Heterogeneous Nuclear Ribonucleoprotein L: Insights into its roles in alternative splicing regulation. J Biol Chem 288: 22636- 22649.

7. Hui J, Stangl K, Lane WS, Bindereif A (2003) HnRNP L stimulates splicing of the eNOS gene by binding to variable-length CA repeats. Nat Struct Biol 10: 33-37.

8. Rossbach O, Hung LH, Schreiner S, Grishina I, Heiner M, et al. (2009) Autoand cross-regulation of the hnRNP $L$ proteins by alternative splicing. Mol Cell Biol 29: 1442-1451.

9. Hung LH, Heiner M, Hui J, Schreiner S, Benes V, et al. (2008) Diverse roles of hnRNP $L$ in mammalian mRNA processing: a combined microarray and RNAi analysis. RNA 14: 284-296.

10. Hermiston ML, Xu Z, Weiss A (2003) CD45: a critical regulator of signaling thresholds in immune cells. Annu Rev Immunol 21: 107-137.

11. Rothrock CR, House AE, Lynch KW (2005) HnRNP L represses exon splicing via a regulated exonic splicing silencer. EMBO J 24: 2792-2802.

12. House AE, Lynch KW (2006) An exonic splicing silencer represses spliceosome assembly after ATP-dependent exon recognition. Nat Struct Mol Biol 13: 937944

13. Chiou NT, Shankarling G, Lynch KW (2013) hnRNP L and hnRNP A1 induce extended U1 snRNA interactions with an exon to repress spliceosome assembly. Mol Cell 49: 972-982.

14. Shankarling G, Lynch KW (2013) Minimal functional domains of paralogues hnRNP $L$ and hnRNP LL exhibit mechanistic differences in exonic splicing repression. Biochem J 453: 271-279.

15. Gaudreau MC, Heyd F, Bastien R, Wilhelm B, Moroy T (2012) Alternative splicing controlled by heterogeneous nuclear ribonucleoprotein $L$ regulates development, proliferation, and migration of thymic pre-T cells. J Immunol 188 : 5377-5388.

16. Yu J, Hai Y, Liu G, Fang T, Kung SK, et al. (2009) The heterogeneous nuclear ribonucleoprotein $L$ is an essential component in the Ca2+/calmodulindependent protein kinase IV-regulated alternative splicing through cytidineadenosine repeats. J Biol Chem 284: 1505-1513.

17. Liu G, Razanau A, Hai Y, Yu J, Sohail M, et al. (2012) A conserved serine of heterogeneous nuclear ribonucleoprotein $L$ ( $($ nRNP L) mediates depolarizationregulated alternative splicing of potassium channels. J Biol Chem 287: 2270922716.

18. Rachel Wilson Goehe JCS, Charuta Murudkar, Sanja Usanovic, Nadia FL, Davis HM, et al. (2010) hnRNP L regulates the tumorigenic capacity of lung cancer xenografts in mice via caspase 9 pre-mRNA processing. J Clin Invest 120: $3923-3939$ 